$\$$

\title{
Hematological effects of benzene in the petroleum industry
}

\author{
Yen-Hsiung Liao \\ Department of Health Sciences, School of Public Health, Kaohsiung Medical University \\ E-mail: bany0309@yahoo.com.tw
}

\begin{abstract}
Purpose: To follow up the hematological changes in petroleum workers with continuous exposure to benzene.

Approach: The hematological data of two petroleum company male employees was obtained from the annual health check-ups during 2001 to 2005. Workers were classified into exposed and unexposed groups on the basis of their exposure operation performed, and the industrial hygiene surveys.

Results: There was not any difference between the exposed and the unexposed groups in the frequency of abnormalities with CBC differential and WBC differential almost. The frequency of higher than the normal range with eosinophils was significantly higher in exposed group $(31.73 \%)$ than in unexposed group $(11.54 \%, \mathrm{p}<0.05)$ in 1994 , only.

Conclusion: The study suggested that the eosinophil counts could be a hematological effect maker among workers exposed to benzene in the petroleum industry.
\end{abstract}

Keywords: Hematological Changes; Benzene; Eosinophil.

\section{Introduction}

Benzene is widely used in the petroleum industry, and known a myelotoxic effect in human (Ert \& Rhoads 1939, Thorpe 1974, Fishbeck et al. 1978, Kipen et al. 1989). Occupational exposure to benzene could decrease the levels of hemoglobin, white cell counts, absolute lymphocyte counts, and red cell counts, mean corpuscular volume, and platelets, as well as increase a risk of leukemia (Fishbeck et al. 1978, Kipen et al. 1989, Ward et al. 1996, Khuder et al. 1999, Duarte-Davidson et al. 2001, Costantini et al. 2003, Rothman et al. 1996). However, some studies suggested that benzene exposure could increase the red blood cell mean corpuscular volume (Fishbeck et al. 1978, Rothman et al. 1996). The demand of benzene in Taiwan occupies at least $2 \%(560 * 106$ ton) over the world. Most of benzene is used in multiple processes in the plastic companies. Workers are susceptible to exposed to benzene by their operation performed.

We selected two petroleum company workers that used the $49.3 \%$ of the benzene in all the Taiwan as the study population. The peripheral blood elements were determined in an attempt to establish whether occupational exposure to benzene may cause a higher prevalence of hematological abnormalities.

\section{Methods}

171 workers from two petroleum companies were classified into the exposed group (production line, and laboratory) and the unexposed group (office workers) on the basis of their exposure operation performed, and the industrial hygiene surveys. The study excluded the 15 female workers because its small sample size.

The hematological data of 156 workers was obtained from the annual health check-ups during 2001 to 2005. Hematological markers included complete blood cell (CBC) differential, and white blood cell (WBC) differential.
The mean age was 36 in the exposed group and 37 in the unexposed group respectively. The mean working year was 7.11 in the exposed group and 6.20 in the unexposed group respectively. There was not any significant difference between the exposed and unexposed group for the mean of their age, and working year in 2001.

All results are presented as the number of low, normal range, high, and unknown. When two groups were compared, the Chi-square test was employed. The limit of significance was set up at $p<$ 0.05 . All analyses were performed with the SPSS program (SPSS Inc., Chicago, IL) for Windows.

\section{Results}

Table 1 shows the distribution of hematological data stratified by exposure condition in petroleum workers. There was not any difference between the exposed and the unexposed groups in the frequency of abnormalities with CBC differential compared from 2001 to 2005 . There was not any difference between the exposed and the unexposed groups in the frequency of abnormalities with neutrophils, lymphocytes, monocytes, and basophils compared from 2001 to 2005 , also. The frequency of abnormalities with eosinophils was significantly higher in exposed group (31.73\%) than in unexposed group $(11.54 \%, \mathrm{p}<0.05)$ in 2004 (Table 2).

Table 1: Hematologic results: $\mathrm{CBC}$ differential in relation to normal range

\begin{tabular}{lrrrrrrrrrr}
\hline & \multicolumn{1}{c}{} & \multicolumn{1}{c}{ Year } \\
& 2001 & \multicolumn{1}{c}{2002} & \multicolumn{2}{c}{2003} & 2004 & 2005 \\
\hline CBC differential & $\mathrm{E}$ & $\mathrm{U}$ & $\mathrm{E}$ & $\mathrm{U}$ & $\mathrm{E}$ & $\mathrm{U}$ & $\mathrm{E}$ & $\mathrm{U}$ & $\mathrm{E}$ & $\mathrm{U}$ \\
& $\mathrm{n}$ & $\mathrm{n}$ & $\mathrm{n}$ & $\mathrm{n}$ & $\mathrm{n}$ & $\mathrm{n}$ & $\mathrm{n}$ & $\mathrm{n}$ & $\mathrm{n}$ & $\mathrm{n}$ \\
WBC & & & & & & & & & & \\
Low & 1 & 1 & 2 & 1 & 3 & 1 & 3 & 1 & 2 & 2 \\
Normal & 99 & 38 & 99 & 37 & 101 & 39 & 91 & 52 & 102 & 38 \\
High & 8 & 1 & 5 & 1 & 9 & 2 & 9 & 0 & 11 & 0 \\
Unknown & 6 & 2 & 7 & 4 & 0 & 1 & 0 & 0 & 1 & 0 \\
Total & 114 & 42 & 113 & 43 & 113 & 43 & 103 & 53 & 116 & 40 \\
\hline
\end{tabular}




\begin{tabular}{|c|c|c|c|c|c|c|c|c|c|c|}
\hline \multicolumn{11}{|l|}{$\mathrm{RBC}$} \\
\hline Low & 5 & 0 & 8 & 3 & 7 & 1 & 6 & 1 & 4 & 0 \\
\hline Normal & 95 & 38 & 92 & 32 & 96 & 37 & 91 & 49 & 102 & 35 \\
\hline High & 9 & 2 & 6 & 4 & 9 & 3 & 7 & 2 & 9 & 5 \\
\hline Unknown & 5 & 2 & 7 & 4 & 2 & 1 & 0 & 0 & 0 & 1 \\
\hline Total & 114 & 42 & 113 & 43 & 114 & 42 & 104 & 52 & 115 & 41 \\
\hline \multicolumn{11}{|l|}{ HGB } \\
\hline Low & 16 & 2 & 8 & 3 & 1 & 0 & 0 & 0 & 0 & 0 \\
\hline Normal & 93 & 38 & 93 & 32 & 103 & 39 & 96 & 50 & 106 & 39 \\
\hline High & 0 & 0 & 2 & 0 & 3 & 1 & 1 & 2 & 8 & 1 \\
\hline Unknown & 5 & 2 & 11 & 7 & 6 & 3 & 7 & 0 & 1 & 1 \\
\hline Total & 114 & 42 & 114 & 42 & 113 & 43 & 104 & 52 & 115 & 41 \\
\hline \multicolumn{11}{|l|}{$\mathrm{HCT}$} \\
\hline Low & 6 & 0 & 7 & 3 & 5 & 1 & 7 & 1 & 2 & 0 \\
\hline Normal & 103 & 40 & 94 & 36 & 106 & 38 & 94 & 45 & 112 & 37 \\
\hline High & 0 & 0 & 0 & 0 & 2 & 2 & 3 & 6 & 1 & 2 \\
\hline Unknown & 5 & 2 & 12 & 4 & 1 & 1 & 0 & 0 & 1 & 1 \\
\hline Total & 114 & 42 & 113 & 43 & 114 & 42 & 104 & 52 & 116 & 40 \\
\hline \multicolumn{11}{|l|}{ MCV } \\
\hline Low & 12 & 0 & 6 & 4 & 8 & 3 & 12 & 1 & 8 & 2 \\
\hline Normal & 96 & 40 & 98 & 35 & 105 & 37 & 92 & 51 & 107 & 37 \\
\hline High & 0 & 0 & 1 & 0 & 0 & 1 & 0 & 0 & 0 & 1 \\
\hline Unknown & 5 & 3 & 9 & 3 & 1 & 1 & 0 & 0 & 0 & 1 \\
\hline Total & 113 & 43 & 114 & 42 & 114 & 42 & 104 & 52 & 115 & 41 \\
\hline \multicolumn{11}{|l|}{ PLT } \\
\hline Low & 3 & 3 & 0 & 2 & 3 & 0 & 2 & 3 & 2 & 2 \\
\hline Normal & 105 & 36 & 103 & 36 & 109 & 40 & 102 & 49 & 111 & 37 \\
\hline High & 0 & 0 & 0 & 0 & 0 & 0 & 0 & 0 & 2 & 1 \\
\hline Unknown & 6 & 3 & 11 & 4 & 3 & 1 & 0 & 0 & 0 & 1 \\
\hline Total & 114 & 42 & 114 & 42 & 115 & 41 & 104 & 52 & 115 & 41 \\
\hline
\end{tabular}

Table 2: Hematologic Results: WBC Differential In Relation To Normal Range

\begin{tabular}{|c|c|c|c|c|c|c|c|c|c|c|}
\hline \multirow{4}{*}{ WBC differential } & \multicolumn{9}{|c|}{ Year } & \\
\hline & \multicolumn{2}{|c|}{2001} & \multicolumn{2}{|c|}{2002} & \multicolumn{2}{|c|}{2003} & \multicolumn{2}{|c|}{2004} & \multicolumn{2}{|c|}{2005} \\
\hline & $\mathrm{E}$ & $\mathrm{U}$ & $\mathrm{E}$ & $\mathrm{U}$ & $\mathrm{E}$ & $\mathrm{U}$ & $E$ & $\mathrm{U}$ & $E$ & $\mathrm{U}$ \\
\hline & $\mathrm{n}$ & $\mathrm{n}$ & $\mathrm{n}$ & $\mathrm{n}$ & $\mathrm{n}$ & $\mathrm{n}$ & $\mathrm{n}$ & $\mathrm{n}$ & $\mathrm{n}$ & $\mathrm{n}$ \\
\hline \multicolumn{11}{|l|}{ Neutrophil } \\
\hline Low & 12 & 2 & 9 & 3 & 10 & 7 & 5 & 0 & 8 & 0 \\
\hline Normal & 85 & 37 & 90 & 35 & 95 & 33 & 80 & 32 & 90 & 39 \\
\hline High & 11 & 1 & 7 & 1 & 7 & 1 & 18 & 20 & 17 & 2 \\
\hline Unknown & 6 & 2 & 7 & 4 & 1 & 2 & 0 & 1 & 0 & 0 \\
\hline Total & 114 & 42 & 113 & 43 & 113 & 43 & 103 & 53 & 115 & 41 \\
\hline \multicolumn{11}{|l|}{ Lymphocyte } \\
\hline Low & 6 & 1 & 4 & 1 & 7 & 1 & 23 & 15 & 8 & 0 \\
\hline Normal & 87 & 37 & 92 & 35 & 92 & 36 & 75 & 37 & 91 & 39 \\
\hline High & 15 & 2 & 10 & 3 & 12 & 5 & 6 & 0 & 16 & 2 \\
\hline Unknown & 6 & 2 & 7 & 4 & 2 & 1 & 0 & 0 & 0 & 0 \\
\hline Total & 114 & 42 & 113 & 43 & 113 & 43 & 104 & 52 & 115 & 41 \\
\hline \multicolumn{11}{|l|}{ Monocyte } \\
\hline Low & 0 & 0 & 2 & 0 & 1 & 0 & 2 & 0 & 1 & 0 \\
\hline Normal & 92 & 36 & 102 & 39 & 111 & 42 & 94 & 50 & 110 & 40 \\
\hline High & 15 & 3 & 2 & 0 & 1 & 0 & 8 & 2 & 4 & 1 \\
\hline Unknown & 7 & 3 & 8 & 3 & 0 & 1 & 0 & 0 & 0 & 0 \\
\hline Total & 114 & 42 & 114 & 42 & 113 & 43 & 104 & 52 & 115 & 41 \\
\hline \multicolumn{11}{|l|}{ Eosinophil } \\
\hline Low & 0 & 0 & 0 & 0 & 2 & 0 & 1 & 0 & 0 & 0 \\
\hline Normal & 106 & 40 & 73 & 33 & 87 & 31 & 67 & $45^{*}$ & * 114 & 40 \\
\hline High & 3 & 0 & 28 & 6 & 18 & 9 & 33 & 6 & 1 & 0 \\
\hline Unknown & 5 & 2 & 12 & 4 & 7 & 2 & 3 & 1 & 1 & 0 \\
\hline Total & 114 & 42 & 113 & 43 & 114 & 42 & 104 & 52 & 116 & 40 \\
\hline \multicolumn{11}{|l|}{ Basophil } \\
\hline Low & 4 & 0 & 0 & 0 & 0 & 0 & 0 & 0 & 11 & 6 \\
\hline Normal & 93 & 36 & 104 & 38 & 110 & 40 & 101 & 51 & 89 & 32 \\
\hline High & 8 & 4 & 1 & 0 & 0 & 0 & 0 & 0 & 15 & 2 \\
\hline Unknown & 7 & 4 & 9 & 4 & 4 & 2 & 3 & 1 & 0 & 1 \\
\hline Total & 112 & 44 & 114 & 42 & 114 & 42 & 104 & 52 & 115 & 41 \\
\hline
\end{tabular}

*: Chi-square test, $\mathrm{p}<0.05$. Normal range: Neutrophils: 45\% - 65\%; Lymphocyte: $25 \%$ - 45\%; Monocyte: $2 \%$ - 8\%; Eosinophils: $1 \%$ - 5\%; Basophils: $0-1 \%$

\section{Discussion}

The study showed elevated eosinophil counts in exposed workers when compared with the unexposed workers. Eosinophils are proposed role in disease processes including infections, asthma, allergic inflammatory response, and anemia (Hogan 2008, p42; Kruse 2008, p8). The presence of the eosinophilia can indicate chronic myelogenous leukemia (Gallin 1991). However, the prevalence of microcytic anemia (MCV $<80 \%$ ) was not correlated with the elevated eosinophils in the work.

We did not see significantly higher frequency of abnormalities with eosinophils in exposed group than in unexposed group in the next year (2005). According to the industrial health practice, such as medical care, and shortening working hours, the change of eosinophils occurred in 2005 expectantly.

The study suggested that the eosinophil counts could be an useful maker among workers exposed to benzene in the petroleum industry. Changes in eosinophils require special attention as a warning sign.

\section{Acknowledgements}

This work was supported by the Jean Tide Medical Examination Center.

\section{References}

[1] Costantini AS, Quinn M, Consonni D, \& Zappa M (2003). Exposure to benzene and risk of leukemia among shoe factory workers. Scan J Work Environ Health 29, 51 - 59. http://dx.doi.org/10.5271/sjweh.704

[2] Duarte-Davidson R, Courage C, Rushton L, \& Levy L (2001). Benzene in the environment: an assessment of the potential risks to the health of the population. Occup Environ Med 58, 2 -13. http://dx.doi.org/10.1136/oem.58.1.2.

[3] Ert LA \& Rhoads CP (1939). The hematological effects of benzene poisoning. J indust Hyg Toxicol 21, $421-435$.

[4] Fishbeck WA, Towmsend JC, \& Swank MG (1978). Effects of chronic occupational exposure to measured concentrations of benzene. Journal of Occupational Medicine 20, 539 - 542. http://dx.doi.org/10.1097/00043764-197808000-00005.

[5] Gallin JL. Disorders of phagocytic cells. In: Wilson JD, Brannwald E, Petersdorf RG, MartinJB, Fauci AS, Root RK, eds. Harrison's Principles of Internal Medicine. 12th Ed. New York: McGraw Hill; 1991:463.

[6] Hogan SP, Rosenberg HF, Moqbel R, Phipps S, Foster PS, Lacy P, Kay AB, \& Rothenberg ME (2008). Eosinophils: Biological properties and role in health and Disease. Clinical \& Experimental Allergy 38 , 709 - 750. http://dx.doi.org/10.1111/j.1365-2222.2008.02958.x.

[7] Khuder SA, Youngdale MC, Bisesi MS, \& Schaub EA (1999). Assessment of complete blood count variations among workers exposed to low levels of benzene. JOEM 41, $821-826$.

[8] Kipen HM, Cody RP, \& Goldstein BD (1989). Use of longitudinal analysis of peripheral blood counts to validate historical reconstructions of benzene exposure. Environmental Health Perspectives 82, 199 - 206. http://dx.doi.org/10.1289/ehp.8982199.

[9] Kruse A, Uehlinger DE, Gotch F. Kotanko P, \& Levin NW. Red blood cell lifespan, erythropoiesis and hemoglobin control. Contrib Nephrol. 161:247-254. http://dx.doi.org/10.1159/000130698.

[10]Rothaman N, Smith MT, Hayes RB, Li GL, Irons RD, Dosemeci M, Haas R, Stillman WS, Linet M, Xi L, Bechtold WE, Wiemels J, Campleman S, Zhang L, Quintana P JE, Titenko-Holland N, Wang YZ, Lu W, Kolachana P, Meyer K, \& Yin S (1996). An epidemiologyic study of early biologic effects of benzene in Chinese workers. Environmental Health Perspectives 104 S6, 1365 - 1368. http://dx.doi.org/10.1289/ehp.961041365.

[11] Thorpe JJ (1974). Epidemiologic survey of leukemia in persons potentially exposed to benzene. J Occup Med 16, 375 - 382

[12]Ward E, Hornung R, Morris J, Rinsky R, Wild D, Halperin W, \& Guthrie W (1996). Risk of low red or white blood cell count related to estimated benzene exposure in a rubberworker cohort $(1940-1975)$ American Journal of Industrial Medicine 29, 247 -257. http://dx.doi.org/10.1002/(SICI)1097-0274(199603)29:3<247::AIDAJIM4>3.0.CO;2-N. 